\title{
Elder Chinese Martial Art Practitioners Have Higher Radial Bone Strength, Hand-Grip Strength, and Better Standing Balance Control
}

\author{
Shirley S. M. Fong, ${ }^{1}$ X. Guo, ${ }^{2}$ Alice P. M. Cheung, ${ }^{2}$ Alex T. L. Jo, ${ }^{2}$ Gary K. W. Lui, ${ }^{2}$ \\ Dennis K. C. Mo, ${ }^{2}$ Shamay S. M. Ng, and William W. N. Tsang ${ }^{2}$ \\ ${ }^{1}$ Institute of Human Performance, The University of Hong Kong, Pokfulam, Hong Kong \\ ${ }^{2}$ Department of Rehabilitation Sciences, The Hong Kong Polytechnic University, Hung Hom, Hong Kong
}

Correspondence should be addressed to Shirley S. M. Fong; smfong@hku.hk

Received 23 May 2013; Accepted 17 June 2013

Academic Editors: A. Danielsson, B. Dugue, J. J. Sosnoff, and C. Zwingmann

Copyright (C) 2013 Shirley S. M. Fong et al. This is an open access article distributed under the Creative Commons Attribution License, which permits unrestricted use, distribution, and reproduction in any medium, provided the original work is properly cited.

\begin{abstract}
This study explored the feasibility of using Ving Tsun (VT) martial art as a fall-prevention exercise for male seniors. We compared the radial bone strength, hand-grip strength, and standing balance control of senior VT practitioners and nonpractitioners under various sensory environments. Sixteen male VT practitioners (mean age \pm SD: $51.3 \pm 7.8$ years) and 17 sex- and age-matched control adults (mean age \pm SD: $53.7 \pm 5.9$ years) participated in the study. The bone strength of the distal radius, hand-grip strength, and standing balance control were assessed using an ultrasound bone sonometer, a Jamar dynamometer, and sensory organization test (SOT) on the NeuroCom Balance Master, respectively. A multivariate analysis of variance (MANOVA) was used to compare the outcome variables between the two groups. Results revealed that VT practitioners had significantly higher radial bone strength ( $T$-score) on the dominant side $(P=0.015)$, hand-grip strength (dominant side, $P=0.034$; nondominant side, $P=0.026$ ), and standing balance control (SOT composite equilibrium score, $P=0.005$ ) than the control participants. Male elder VT practitioners had higher radial bone strength, hand-grip strength, and better standing balance control than nonpractitioners. Our findings shed light on the use of VT exercise to prevent falls and fall-related fractures of the distal radius in male elders.
\end{abstract}

\section{Introduction}

Falls among the elderly are common and a global cause of morbidity and mortality. It has been reported that there are over 400 potential risk factors for falling [1]. Among these risk factors, the physical deteriorations associated with advancing age such as decreased bone mass [2], decreased muscular strength [3, 4], and balance deficits [5], along with physical inactivity [6-8], have been found to be the major determinants of falls among seniors [1]. Improving bone and muscular strength and postural stability using exercises could reduce fall risks and fall-related injuries (e.g., the fracture of the distal radius) $[9,10]$, improving the quality of life experienced by the elderly $[8,11]$.

To date, ample studies have investigated the effects of fallprevention programs (e.g., Tai Chi) on improving physical impairments (e.g., decreased bone and muscular strength), functioning (e.g., body balance) and reducing falls or fall risk among elderly women [12-15]. However, elderly men are an underexamined group that also suffers from falls and subsequent osteoporotic fractures. Studies have been called for to design treatment strategies specifically for elderly men [16].

Ving Tsun (VT) is a traditional Chinese martial art characterized by rapid and forceful punching techniques and stable yet agile footwork. Owing to its simplicity and functionality as self-defense, VT is popular among male adults all over the world. The most well-known practitioners of VT were Bruce Lee and Ip Man [17-19]. VT practitioners are considered physically strong because they undergo highimpact training such as wooden dummy training (Figure 1), sticking-hand exercise (Figure 2), and sandbag workout with "one-inch punch" $[18,19]$. We hypothesized that VT practitioners' forearm bone and muscle strength and body balance might be superior to those of nonpractitioners. However, 


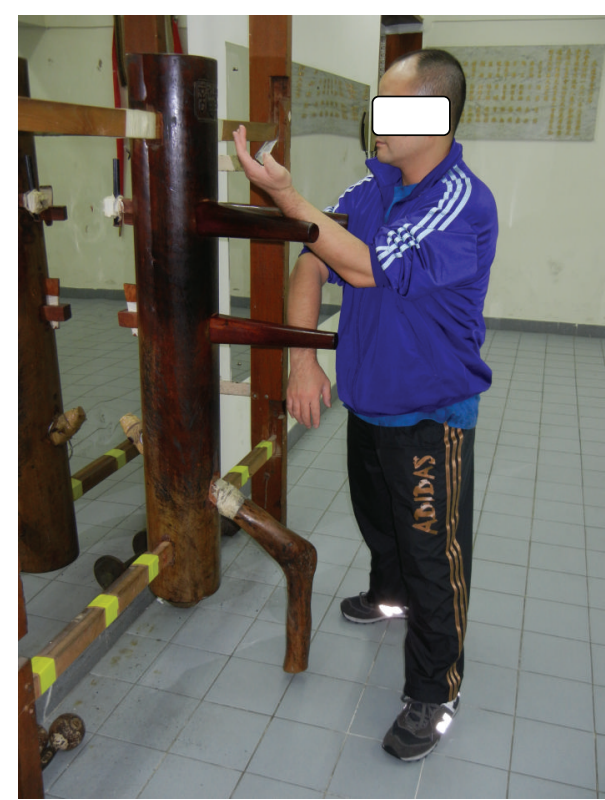

Figure 1: Ving Tsun wooden dummy training.

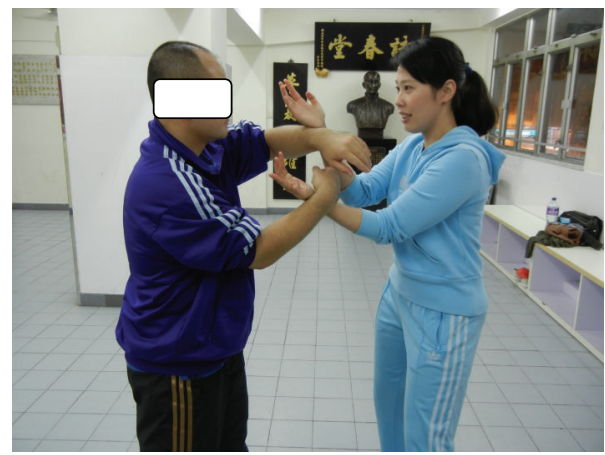

FIgURE 2: Ving Tsun sticking-hand drills.

to our knowledge, no study has investigated the beneficial effects of VT training in male seniors thus far.

The aims of this pilot study were to (1) compare the radial bone strength, hand-grip strength, and standing balance control between senior male VT practitioners and nonpractitioners under various sensory conditions and (2) examine whether there were any side-to-side differences in forearm bone and muscle strength among VT practitioners.

\section{Materials and Methods}

2.1. Participants. Thirty-three male older adults participated in the study, 16 of whom were advanced VT practitioners. They were recruited from three local VT schools by convenience sampling, and each had over three years of VT experience, trained for two to eight hours per week, and had attained the instructor level. The other 17 participants were healthy seniors recruited from the community. The participant characteristics are described in Table 1. None of the participants engaged in any regular physical training
TABLE 1: Participants' characteristics.

\begin{tabular}{lccc}
\hline & $\begin{array}{c}\text { VT group } \\
(\text { mean } \pm \text { SD }) \\
(n=16)\end{array}$ & $\begin{array}{c}\text { Control group } \\
(\text { mean } \pm \text { SD }) \\
(n=17)\end{array}$ & $P$ value \\
\hline Age, years & $51.3 \pm 7.8$ & $53.7 \pm 5.9$ & 0.315 \\
Sex, $n$ & 16 males & 17 males & \\
Height, m & $1.69 \pm 0.05$ & $1.68 \pm 0.06$ & 0.585 \\
Weight, kg & $73.0 \pm 9.3$ & $72.0 \pm 8.1$ & 0.747 \\
$\begin{array}{l}\text { Ving Tsun experience, } \\
\text { years }\end{array}$ & $16.1 \pm 9.6$ & 0 & \\
\hline
\end{tabular}

(except VT training for the VT group participants), and any who exhibited significant cognitive, musculoskeletal, cardiopulmonary, visual, vestibular, or neurological disorders that might affect physical performance were excluded. This study was approved by the Human Subjects Ethics Review Subcommittee of the Hong Kong Polytechnic University. The testing procedures were fully explained to the participants, and written consent was obtained before data collection.

2.2. Procedures and Measures. Data collection was performed by a physiotherapist and four physiotherapy students between June 2012 and February 2013 following Declaration of Helsinki guidelines. All of the assessments took place in the Sports Training and Rehabilitation Laboratory of the Hong Kong Polytechnic University. Relevant information such as general health and exercise habits was obtained by interviewing the participants face to face. The body height and weight of each participant were first measured by a mechanical scale equipped with a height rod (420KL Physician Scale, HealthO-Meter, Bridgeview, IL, USA). Participants then underwent the following assessments in random order.

2.2.1. Bone Strength of Distal Radius. The bone strength of the distal radius was assessed using the Sunlight Mini-Omni Ultrasound Bone Sonometer (Sunlight, BeamMed Ltd., Israel) because it is radiation free and the measurement is independent of body height and weight [20]. Ultrasound gel was applied to the skin surface at the measurement site to facilitate acoustic coupling. Placement of the handheld probe was at the distal third of the radius (i.e., midpoint between the olecranon process of the ulna and the tip of the distal phalanx of the third digit). The transducers within the probe were rotated around the radius slowly by the assessor without lifting the probe from the skin surface. The measurement was repeated at least three times. The velocity of the ultrasound wave ("speed of sound" or "SOS" in $\mathrm{m} / \mathrm{s}$ ) traveling through a few centimeters of radial bone parallel to its axis within the outer $2 \mathrm{~mm}$ to $6 \mathrm{~mm}$ was measured [21]. The SOS values were then converted into a $T$-score using inbuilt computer software. The SOS $T$-score represents the units of standard deviations relative to population reference values of healthy young adults of the same ethnicity. It reflects the bone's fragility and is highly correlated with bone strength [21, 22]. However, unlike the $T$-score obtained by using dualenergy X-ray absorptiometry, SOS measurements cannot 
predict forearm bone mineral density [22], and hence the standard World Health Organization's (WHO) definition of osteoporosis cannot be applied in this case. Instead, SOS Tscore thresholds for diagnosis of osteoporosis and osteopenia at the distal radius are suggested to be -2.6 and -1.4 , respectively [23]. The average SOS $T$-scores of the three trials (obtained from both dominant and nondominant arms) were used for analysis. The Sunlight ultrasound systems have been reported to be reliable (intraoperator precision at distal radius: $0.36 \%$ ) and precise (in vivo precision: $0.4 \%-0.8 \%$ ) in previous studies [23-25].

2.2.2. Hand-Grip Strength. All of the participants were assessed for maximal hand-grip strength using a Jamar dynamometer (Sammons Preston, Mississauga, ON, Canada). Standard assessment procedures were followed [26]. In brief, participants were in a standing position with the testing shoulder adducted and neutrally rotated, elbow flexed at $90^{\circ}$, forearm in midprone, and wrist in a neutral position. Before the actual tests, the participants were allowed to squeeze the dynamometer twice to familiarize themselves with the testing procedure. This was followed by a 10 -second rest. Participants were then instructed to squeeze the dynamometer using maximal effort. A total of three trials were performed on each side, and the highest grip-strength value of each hand was recorded [26]. The interrater reliability (ICC: 0.98) and intrarater reliability (ICC: 0.94-0.98) of this hand-grip strength test were reported to be good to excellent [26].

2.2.3. Postural Control. Standing balance was assessed using the sensory organization test (SOT) performed on the NeuroCom Balance Master (Smart Equitest, NeuroCom International Inc., Clackamas, OR, USA). This machine quantifies the participants' ability to maintain standing balance under reduced or conflicting sensory conditions. The participants stood barefoot on the movable platform of the machine and wore a security harness to prevent falling. They were then instructed to maintain bipedal standing balance under six sensory conditions that provide inaccurate or absent somatosensory, visual, or vestibular information to the participant. Each condition consisted of three trials and each trial lasted for 20s. The six conditions included (1) eyes open, stable visual surround and platform; (2) eyes closed, stable visual surround and platform; (3) eyes open, sway-referenced visual surround and stable platform; (4) eyes open, stable visual surround and sway-referenced platform; (5) eyes closed, stable visual surround and sway-referenced platform; and (6) eyes open, sway-referenced visual surround and platform. All of the participants were given a familiarization trial before the actual test [27].

The platform (dual-force plates) recorded the trajectory of the participant's center of pressure (COP) during postural sway. The NeuroCom computer program then generated an equilibrium score (ES) for each of the six sensory conditions based on the COP movements in standing. Moreover, a composite ES, which characterized the overall level of performance, was calculated from the weighted average of the ES of conditions 1 through 6. Higher ES (close to 100) represents little sway in standing, whereas lower ES (close to 0 ) represents excessive sway that may exceed the limit of stability $[27,28]$. The ES of each sensory condition and the composite ES were used for analysis. A previous study showed that the ICC values of the six sensory conditions ranged from 0.72 to 0.93 , indicating moderate-to-good testretest reliability of SOT in older adults [15].

2.3. Statistical Analysis. The normal distribution of outcome variables was first checked using the Kolmogorov-Smirnov test. Independent $t$-tests were used to compare the age, body height, and weight between the VT and control groups. To compare the bone strength of the distal radius and the handgrip strength between the two groups, a single multivariate analysis of variance (MANOVA) incorporating all relevant outcomes (i.e., SOS $T$-scores of bilateral radii and bilateral hand-grip strength values) was performed. Another separate MANOVA was conducted to compare the SOT-derived outcomes (i.e., equilibrium scores of conditions 1 to 6 ) between the two groups. Because the composite ES is the average of all of the equilibrium scores, an independent $t$-test was used to identify whether there was any significant between-group difference for this outcome parameter. The results from the MANOVA showed the effects of the group on all outcomes simultaneously and the corresponding Bonferroni-adjusted $P$ values, hence avoiding the increased probability of type I errors. Effect sizes (i.e., partial eta-squared for MANOVA and Cohen's d for independent $t$-test) are also reported for each outcome variable. By convention, Cohen's $d$ values of $0.2,0.5$, and 0.8 are considered small, medium, and large effect sizes, respectively. For partial eta-squared values, 0.01 , 0.06 , and 0.14 represent small, medium, and large effect sizes, respectively [29]. Secondary data analysis was carried out to further examine whether there were any side-to-side differences in SOS $T$-score and hand-grip strength values in the VT group. All of the previous statistical analyses were performed using the IBM Statistical Package for Social Sciences 20.0 software. A significance level of 0.05 was set for the statistical tests (two tailed).

Because the sample size was relatively small, it was necessary to determine whether our study was underpowered to detect significant differences between groups. Post hoc power analyses were performed to examine the statistical power of the comparisons of radial bone strength, hand-grip strength, and SOT-derived scores between the VT and control groups. The G Power computer program (Faul \& Erdfelder, Bonn University, Germany) was used.

\section{Results}

There was no significant difference between the two groups in age, body height, or weight (Table 1). Regarding the bone strength of the distal radius, the SOS $T$-score obtained from the dominant side was significantly higher in the VT participants than in the control participants by $53.8 \%(P=0.015)$. However, the SOS $T$-score obtained from the nondominant side showed no significant difference between the two groups $(P=0.093)$ (Table 2$)$. Secondary analysis demonstrated 
TABLE 2: Between-group comparison of radial bone strength, hand-grip strength, and balance control.

\begin{tabular}{|c|c|c|c|c|}
\hline & VT group $($ mean $\pm \mathrm{SD})(n=16)$ & Control group (mean $\pm \mathrm{SD})(n=17)$ & $P$ value & Effect size \\
\hline \multicolumn{5}{|c|}{ Bone strength of distal radius (SOS $T$-score) } \\
\hline Dominant side & $-0.6 \pm 0.8^{\dagger}$ & $-1.3 \pm 0.8$ & $0.015^{*}$ & 0.176 \\
\hline Nondominant side & $-1.0 \pm 1.0$ & $-1.6 \pm 1.0$ & 0.093 & 0.088 \\
\hline \multicolumn{5}{|l|}{ Hand-grip strength } \\
\hline Dominant side, $\mathrm{kg}$ & $42.1 \pm 7.0^{\dagger}$ & $37.1 \pm 6.0$ & $0.034^{*}$ & 0.136 \\
\hline Nondominant side, $\mathrm{kg}$ & $39.7 \pm 4.5$ & $35.7 \pm 5.2$ & $0.026^{*}$ & 0.149 \\
\hline \multicolumn{5}{|l|}{ Sensory organization test } \\
\hline Condition $1 \mathrm{ES}$ & $94.9 \pm 1.3$ & $92.5 \pm 4.1$ & $0.037^{*}$ & 0.137 \\
\hline Condition 2 ES & $91.4 \pm 4.2$ & $91.9 \pm 2.3$ & 0.703 & 0.005 \\
\hline Condition 3 ES & $92.0 \pm 3.5$ & $89.4 \pm 4.9$ & 0.083 & 0.097 \\
\hline Condition 4 ES & $81.9 \pm 8.1$ & $75.9 \pm 9.8$ & 0.067 & 0.107 \\
\hline Condition 5 ES & $62.6 \pm 12.8$ & $53.9 \pm 16.8$ & 0.108 & 0.084 \\
\hline Condition 6 ES & $68.7 \pm 10.7$ & $54.7 \pm 14.2$ & $0.004^{*}$ & 0.247 \\
\hline Composite ES & $78.7 \pm 5.4$ & $71.7 \pm 7.5$ & $0.005^{*}$ & 0.233 \\
\hline
\end{tabular}

${ }^{*}$ Denotes a significant between-group difference $(P<0.05)$.

${ }^{\dagger}$ Denotes a difference significant at $P<0.05$ when compared to the nondominant side.

that the SOS T-score of the dominant side was significantly higher than that of the non-dominant side among the VT practitioners $(P=0.044)$ (Table 2$)$.

The grip-strength values of both dominant and non-dominant hands were higher in the VT group by $13.5 \%(P=0.034)$ and $11.2 \%(P=0.026)$, respectively, than in the control group (Table 2). Additionally, the dominant hand demonstrated significantly higher hand-grip strength than the non-dominant hand $(P=0.010)$ (Table 2$)$. For the SOT-derived scores, between-group differences were found in the ES of conditions $1(P=0.037)$ and $6(P=0.004)$ and the composite ES $(P=0.005)$. No significant difference was shown in the ES of SOT conditions 2 to 5 . For the outcome variables that showed nonsignificant between-group differences, the partial eta-squared values ranged from 0.084 to 0.107 , indicating medium-to-large effect sizes (except for the ES of SOT condition 2) (Table 2).

Post hoc power analysis showed that the statistical powers for the comparisons of radial bone strength (SOS $T$-score) between the VT and control groups were 0.68 (dominant side) and 0.39 (non-dominant side). For the between-group comparisons of hand-grip strength, the statistical powers were 0.57 (dominant side) and 0.63 (non-dominant side). For the comparisons of SOT-derived ES between the two groups, statistical powers were low, ranging from 0.07 to 0.59 for SOT conditions 1 to 5, but high for SOT condition 6 (0.87) and for the composite ES (0.85). This may explain why the ES of SOT conditions 2 to 5 failed to show a significant between-group difference in the MANOVA analysis (Table 2).

\section{Discussion}

This is the first study to investigate bone strength in older VT practitioners. Our results show that the bone strength of the distal radius (dominant side) was significantly higher in the VT practitioners than among the control participants. This finding was anticipated because VT training, which includes wooden dummy training (Figure 1), stickinghand drills (Figure 2), and sandbag workouts, involves many upper limb striking movements that load the bones in the forearms with repeated high peak forces and impacts [18]. According to Wolff's law of bone remodeling, the internal structure of bone is adapted to mechanical demands such that the trabecular patterns (i.e., orientation of trabeculae) coincide with stress trajectories. That is, if loading on a bone increases, the bone will remodel to become stronger to resist that particular type of load [30]. Previous studies have reported that athletes who engage in high-impact sports such as karate display higher bone mineral density in specific regions than sedentary controls [31]. We postulated that longterm regular high-impact VT training might improve radial bone strength, therefore minimizing the risk of distal radius fractures in elderly males [32].

Despite the positive finding on the dominant side, our results demonstrated no between-group difference in radial bone strength on the non-dominant side with a medium effect size of 0.088 . Secondary analysis showed that radial bone strength on the non-dominant side was actually $66.7 \%$ lower than that of the dominant side. This may be because fewer striking movements are performed with the left arm during wooden dummy training [18]. In addition, VT practitioners might use their non-dominant arm less frequently than their dominant arm during dynamic sticking-hand exercise or even during daily activities [33]. Therefore, the mechanical stresses placed on the non-dominant radius were decreased, and the bone became weaker as a result [30].

Another encouraging finding of this study is that VT practitioners demonstrated higher hand-grip strength, especially on the dominant side, than the control participants. This may be attributed to the repeated practice of punching (e.g., vertical fist or "one-inch punch") and grasping (e.g., grappling-hand) techniques during VT training [18, 19]. Extrinsic and intrinsic hand muscles might have been strengthened through these kinds of gripping exercises. 
Our present finding actually concurs with a previous study that showed a significant improvement in hand-grip strength after Tai Chi training. The authors proposed that contraction and relaxation of forearm muscles might increase the muscle tone and hand-grip strength [34]. Previous studies have also suggested that grip strength is independently associated with radial bone mineral density $[35,36]$. Perhaps higher handgrip strength and stronger forearm muscles contribute to the higher radial bone strength among the VT practitioners. Further study might fruitfully explore the relationships between grip strength, forearm bone mineral density, and bone mineral content among VT practitioners.

VT practitioners seem to have better standing balance control under various sensory conditions, as demonstrated by their higher ES (SOT conditions 1 and 6) and composite ES. Although there were no significant differences between groups in the ES of SOT conditions 3 to 5, the effect sizes were moderate to large (0.084-0.107), indicating that the insignificant results might simply be due to the decreased statistical power (0.37-0.50). We might be able to detect significant differences between the two groups if the sample size was increased to 752 participants per group. For the ES of SOT condition 2 , the effect size was exceptionally small (0.005) together with a large $P$ value $(P=0.703)$. We postulated that VT practitioners may not be superior to their sedentary counterparts when standing with eyes closed. Indeed, training with eyes closed is not common during VT practice [19]. VT practitioners are conditioned to maintain postural stability under normal (i.e., SOT condition 1), reduced, and conflicting sensory environments (SOT conditions 3 to 6) instead.

One possible reason for the better standing balance (i.e., less postural sway) in VT practitioners under normal sensory environment (i.e., SOT condition 1) may be the tremendous time they spent in practicing static balance control. VT practitioners practice the basic "Siu Nim Tao" form in the static "goat-gripping" stance during every training session because it is the foundation of all VT techniques [19]. Practitioners are required to maintain postural stability (i.e., minimize postural sway) during different upper limb movements, and this may have trained the static and anticipatory postural control systems of the practitioners $[19,37]$. If this is so, then why did VT practitioners sway less than the control participants when standing under sensory-depriving and conflicting environments? Perhaps VT practitioners develop better visual (reflected by the ES of SOT condition 4) and vestibular (reflected by the ES of SOT condition 5) senses for postural control $[27,28]$ through the dynamic sticking-hand exercise [19]. Further study is needed to explore the mechanisms through which VT practice could improve postural control under various sensory environments.

Our present findings shed light on the use of VT exercise to prevent fall and fall-related fractures of the distal radius in male elders. However, it is not definitive whether participating in VT lessons would increase one's exposure to risk of falling and fracture. The advanced VT practitioners in our study started VT training at a relatively young age (average 35.2 years old). Therefore, it is advisable to start VT training early, preferably before the deterioration of balance ability [5] and before the onset of osteopenia [2]. For people who are receiving VT training, our findings hint that they could exercise both upper limbs in a more symmetrical manner so that their bilateral radii, forearm, and hand muscles might be strengthened.

This study has several limitations. First, the small sample size may have compromised the statistical power. This could explain some of our insignificant findings. Second, it was a cross-sectional study. No causal relationship between practicing VT and the aforementioned beneficial effects could be established. Third, our participants were exclusively in their fifties. They were not frail elders with high fall or fracture risks. Further studies could consider using a randomized controlled study design that includes a larger sample of older males. Moreover, it may be necessary to modify the traditional VT training to suit the weaker participants.

\section{Conclusions}

Male elder VT practitioners had higher radial bone strength, hand-grip strength, and better standing balance control than nonpractitioners. Our findings may inspire the development of VT exercise as an evidence-based medium for preventing fall and fall-related fractures in male seniors.

\section{Conflict of Interests}

The authors declare that they have no conflict of interests with respect to the authorship or publication of this paper. No funding was provided for its preparation.

\section{Acknowledgments}

The authors thank Mr. Alex Ngai, Mr. Sunny So, Mr. Fung Man, and Mr. So Yip for their advice on Ving Tsun martial art and the recruitment of participants.

\section{References}

[1] T. Masud and R. O. Morris, "Epidemiology of falls," Age and Ageing, vol. 30, no. 4, pp. 3-7, 2001.

[2] E. M. Haney and M. M. Bliziotes, "Male osteoporosis: new insights in an understudied disease," Current Opinion in Rheumatology, vol. 20, no. 4, pp. 423-428, 2008.

[3] R. S. Lindle, E. J. Metter, N. A. Lynch et al., "Age and gender comparisons of muscle strength in 654 women and men aged 20-93 yr," Journal of Applied Physiology, vol. 83, no. 5, pp. 15811587, 1997.

[4] B. K. S. Chan, L. M. Marshall, K. M. Winters, K. A. Faulkner, A. V. Schwartz, and E. S. Orwoll, "Incident fall risk and physical activity and physical performance among older men: the osteoporotic fractures in men study," American Journal of Epidemiology, vol. 165, no. 6, pp. 696-703, 2007.

[5] P. W. Overstall, A. N. Exton Smith, F. J. Imms, and A. L. Johnson, "Falls in the elderly related to postural imbalance," British Medical Journal, vol. 1, no. 6056, pp. 261-264, 1977.

[6] S. R. Lord, J. A. Ward, P. Williams, and K. J. Anstey, "An epidemiological study of falls in older community-dwelling women: the Randwick falls and fractures study," Australian Journal of Public Health, vol. 17, no. 3, pp. 240-245, 1993. 
[7] J. S. W. Lee, T.-W. Auyeung, T. Kwok, E. M. C. Lau, P.-C. Leung, and J. Woo, "Associated factors and health impact of sarcopenia in older Chinese men and women: a cross-sectional study," Gerontology, vol. 53, no. 6, pp. 404-410, 2008.

[8] S. Khosla, S. Amin, and E. Orwoll, "Osteoporosis in men," Endocrine Reviews, vol. 29, no. 4, pp. 441-464, 2008.

[9] R. G. Crilly, L. Delaquerriere Richardson, and J. H. Roth, "Postural stability and Colles' fracture," Age and Ageing, vol. 16, no. 3, pp. 133-138, 1987.

[10] J. L. Kelsey, M. M. Prill, T. H. M. Keegan et al., "Reducing the risk for distal forearm fracture: preserve bone mass, slow down, and don't fall!", Osteoporosis International, vol. 16, no. 6, pp. 681690, 2005.

[11] C. P. Iglesias, A. Manca, and D. J. Torgerson, "The health-related quality of life and cost implications of falls in elderly women," Osteoporosis International, vol. 20, no. 6, pp. 869-878, 2009.

[12] A. J. Campbell, M. C. Robertson, M. M. Gardner, R. N. Norton, M. W. Tilyard, and D. M. Buchner, "Randomised controlled trial of a general practice programme of home based exercise to prevent falls in elderly women," British Medical Journal, vol. 315, no. 7115, pp. 1065-1069, 1997.

[13] S. E. Sihvonen, S. Sipilä, and P. A. Era, "Changes in postural balance in Frail Elderly women during a 4-week visual feedback training: a randomized controlled trial," Gerontology, vol. 50, no. 2, pp. 87-95, 2004.

[14] J. O. Judge, C. Lindsey, M. Underwood, D. Winsemius, and E. A. Keshner, "Balance improvements in older women: effects of exercise training," Physical Therapy, vol. 73, no. 4, pp. 254-265, 1993.

[15] W. W. Tsang, V. S. Wong, S. N. Fu, and C. W. Hui-Chan, "Tai Chi improves standing balance control under reduced or conflicting sensory conditions," Archives of Physical Medicine and Rehabilitation, vol. 85, no. 1, pp. 129-137, 2004.

[16] L. J. Melton III, E. J. Atkinson, M. K. O’Connor, W. M. O’Fallon, and B. L. Riggs, "Bone density and fracture risk in men," Journal of Bone and Mineral Research, vol. 13, no. 12, pp. 1915-1923, 1998.

[17] T. A. Green, Martial Arts of the World: An Encyclopedia, ABCCLIO, Goleta, Calif, USA, 2001.

[18] C. Yip, 116 Wing Tsun Dummy Techniques as Demonstrated by grandmaster Yip Man, Leung's Publications, Hong Kong, China, 1981.

[19] D. Peterson, Look Beyond the Pointing Finger-the Combat Philosophy of Wong Shun Leung, Melbourne Chinese Martial Arts Club, Victoria, Australia, 2001.

[20] Z. Zadik, D. Price, and G. Diamond, "Pediatric reference curves for multi-site quantitative ultrasound and its modulators," Osteoporosis International, vol. 14, no. 10, pp. 857-862, 2003.

[21] BeamMed Ltd, Sunlight Miniomni Bone Sonometer User Guide, BeamMed, Petach-Tikva, Israel, 2010.

[22] D. Hans, A. M. Schott, and P. J. Meunier, "Ultrasonic assessment of bone: a review," European Journal of Medicine, vol. 2, no. 3, pp. 157-163, 1993.

[23] K. M. Knapp, G. M. Blake, T. D. Spector, and I. Fogelman, "Multisite quantitative ultrasound: precision, age- and menopauserelated changes, fracture discrimination, and T-score equivalence with dual-energy X-ray absorptiometry," Osteoporosis International, vol. 12, no. 6, pp. 456-464, 2001.

[24] R. Barkmann, E. Kantorovich, C. Singal et al., "A new method for quantitative ultrasound measurements at multiple skeletal sites: first results of precision and fracture discrimination," Journal of Clinical Densitometry, vol. 3, no. 1, pp. 1-7, 2000.
[25] W. M. Drake, M. McClung, C. F. Njeh et al., "Multisite bone ultrasound measurement on North American female reference population," Journal of Clinical Densitometry, vol. 4, no. 3, pp. 239-248, 2001.

[26] A. Peolsson, R. Hedlund, and B. Oberg, "Intra- and inter-tester reliability and reference values for hand strength," Journal of Rehabilitation Medicine, vol. 33, no. 1, pp. 36-41, 2001.

[27] NeuroCom, Balance Manager Systems-Instructions for Use, NeuroCom International, Clackamas, Ore, USA, 2008.

[28] L. M. Nashner, "Computerized dynamic posturography," in Handbook of Balance Function and Testing, Mosby Year book, St. Louis, Mo, USA, 1997.

[29] L. G. Portney and M. P. Watkins, Foundations of Clinical Research-Applications to Practice, Pearson Education, Cranbury, NJ, USA, 2009.

[30] C. Ruff, B. Holt, and E. Trinkaus, "Who's afraid of the big bad Wolff?: "Wolff's law" and bone functional adaptation," American Journal of Physical Anthropology, vol. 129, no. 4, pp. 484498, 2006.

[31] A. Andreoli, M. Monteleone, M. Van Loan, L. Promenzio, U. Tarantino, and A. De Lorenzo, "Effects of different sports on bone density and muscle mass in highly trained athletes," Medicine and Science in Sports and Exercise, vol. 33, no. 4, pp. 507-511, 2001.

[32] Y. Jiang, J. Zhao, C. Rosen, P. Geusens, and H. K. Genant, "Perspectives on bone mechanical properties and adaptive response to mechanical challenge," Journal of Clinical Densitometry, vol. 2, no. 4, pp. 423-433, 1999.

[33] L. B. Bagesteiro and R. L. Sainburg, "Handedness: dominant arm advantages in control of limb dynamics," Journal of Neurophysiology, vol. 88, no. 5, pp. 2408-2421, 2002.

[34] K.-M. Chen, J.-N. Lin, H.-S. Lin et al., "The effects of a Simplified Tai-Chi Exercise Program (STEP) on the physical health of older adults living in long-term care facilities: a single group design with multiple time points," International Journal of Nursing Studies, vol. 45, no. 4, pp. 501-507, 2008.

[35] M. Di Monaco, R. Di Monaco, M. Manca, and A. Cavanna, "Handgrip strength is an independent predictor of distal radius bone mineral density in postmenopausal women," Clinical Rheumatology, vol. 19, no. 6, pp. 473-476, 2000.

[36] S. Tsuji, N. Tsunoda, H. Yata, F. Katsukawa, S. Onishi, and H. Yamazaki, "Relation between grip strength and radial bone mineral density in young athletes," Archives of Physical Medicine and Rehabilitation, vol. 76, no. 3, pp. 234-238, 1995.

[37] A. Shumway-Cook and M. H. Woollacott, "Development of postural control," in Motor Control Translating Research Into Clinical Practice, A. Shumway-Cook and M. H. Woollacott, Eds., Lippincott Williams and Wilkins, Philadelphia, Pa, USA, 2007. 


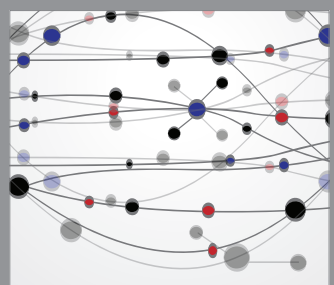

The Scientific World Journal
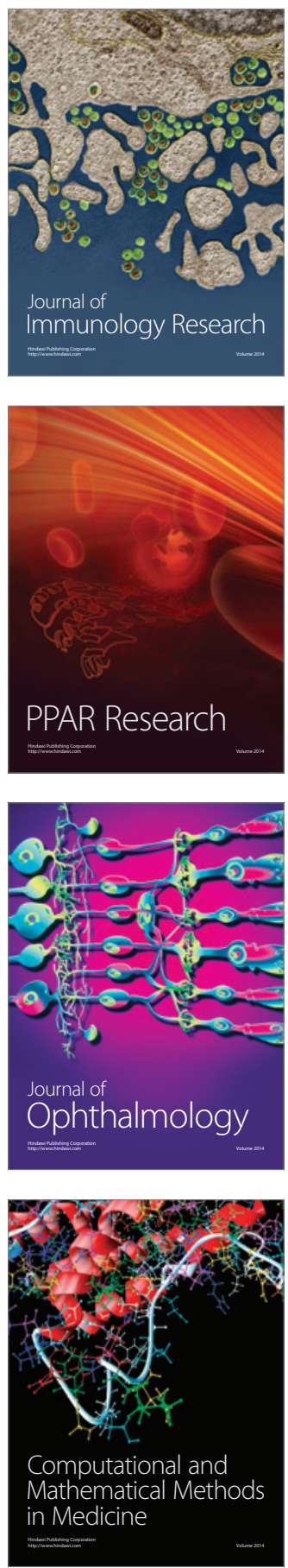

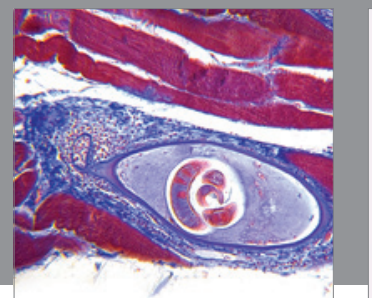

Gastroenterology

Research and Practice
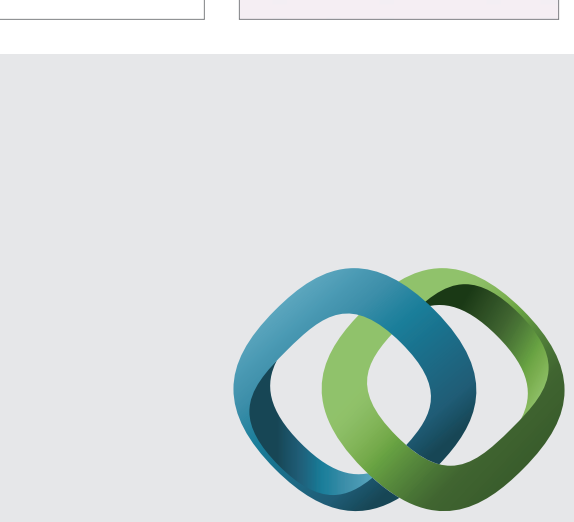

\section{Hindawi}

Submit your manuscripts at

http://www.hindawi.com
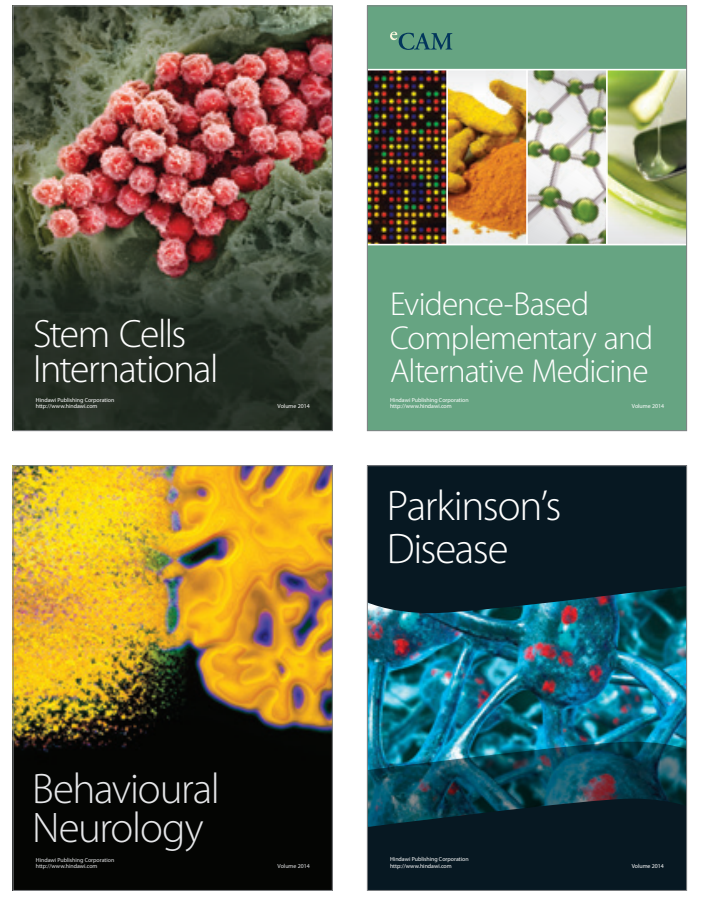
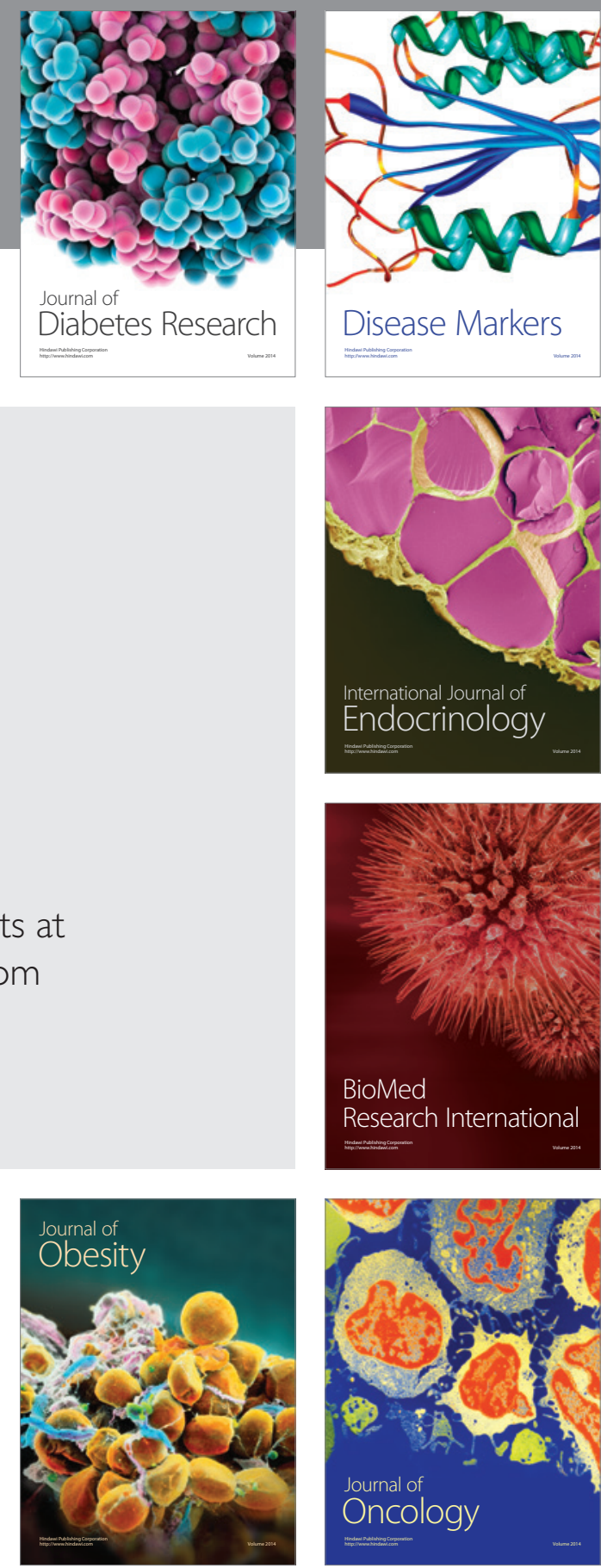

Disease Markers
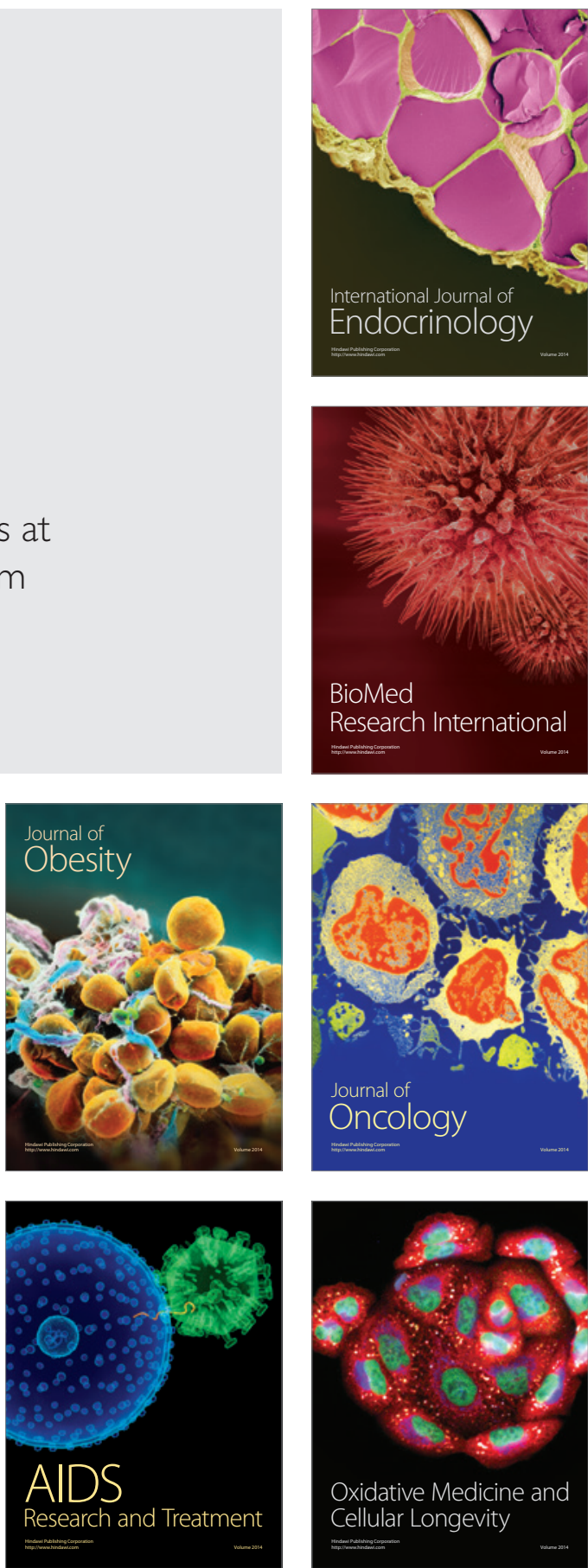